\title{
Independent intrachromosomal recombination events underlie the pericentric inversions of chimpanzee and gorilla chromosomes homologous to human chromosome 16
}

\author{
Violaine Goidts, ${ }^{1}$ Justyna M. Szamalek, ${ }^{1}$ Pieter J. de Jong, ${ }^{2}$ David N. Cooper, ${ }^{3}$ \\ Nadia Chuzhanova, ${ }^{3,4}$ Horst Hameister, ${ }^{1}$ and Hildegard Kehrer-Sawatzki ${ }^{1,5}$ \\ ${ }^{1}$ Department of Human Genetics, University of UIm, 89081 UIm, Germany; ${ }^{2}$ Children's Hospital Oakland Research Institute, \\ Oakland, California 94609, USA; ${ }^{3}$ Institute of Medical Genetics and ${ }^{4}$ Biostatistics and Bioinformatics Unit, Cardiff University, \\ Heath Park, Cardiff, CF14 4XN, United Kingdom
}

\begin{abstract}
Analyses of chromosomal rearrangements that have occurred during the evolution of the hominoids can reveal much about the mutational mechanisms underlying primate chromosome evolution. We characterized the breakpoints of the pericentric inversion of chimpanzee chromosome 18 (PTR XVI), which is homologous to human chromosome 16 (HSA 16). A conserved 23-kb inverted repeat composed of satellites, LINE and Alu elements was identified near the breakpoints and could have mediated the inversion by bringing the chromosomal arms into close proximity with each other, thereby facilitating intrachromosomal recombination. The exact positions of the breakpoints may then have been determined by local DNA sequence homologies between the inversion breakpoints, including a 22-base pair direct repeat. The similarly located pericentric inversion of gorilla (GGO) chromosome XVI, was studied by FISH and PCR analysis. The p- and q-arm breakpoints of the inversions in PTR XVI and GGO XVI were found to occur at slightly different locations, consistent with their independent origin. Further, FISH studies of the homologous chromosomal regions in macaque and orangutan revealed that the region represented by HSA BAC RP11-696P19, which spans the inversion breakpoint on HSA 16q11-12, was derived from the ancestral primate chromosome homologous to HSA 1 . After the divergence of orangutan from the other great apes $\sim 12$ million years ago (Mya), a duplication of the corresponding region occurred followed by its interchromosomal transposition to the ancestral chromosome 16q. Thus, the most parsimonious interpretation is that the gorilla and chimpanzee homologs exhibit similar but nonidentical derived pericentric inversions, whereas HSA 16 represents the ancestral form among hominoids.
\end{abstract}

[Supplemental material is available online at www.genome.org. The sequence data from this study have been submitted to GenBank under accession nos. AY822675, AY822676, and AY822677.]

A variety of different types of genetic change are thought to be responsible for the process of speciation, including gross chromosomal rearrangements and, at least potentially, gene diversification in terms of structure, copy number, and also expression (Taylor et al. 2001; Nahon 2003; Hey 2003; Wu and Ting 2004). Comparative analysis of the chimpanzee (Pan troglodytes, PTR) and human (Homo sapiens, HSA) genomes should potentiate the identification of the key events that have taken place during human chromosomal evolution at the same time as providing new insights into the underlying mutational mechanisms that have fashioned extant primate genomes. The study of these evolutionary changes may also shed some light on hominid evolution that occurred under parapatric conditions in East Africa (Marques-Bonet et al. 2004; Vallender and Lahn 2004; Zhang et al. 2004).

\section{${ }^{5}$ Corresponding author.}

E-mail hildegard.kehrer-sawatzki@medizin.uni-ulm.de; fax 49731 $5002-3438$.

Article and publication are at http://www.genome.org/cgi/doi/10.1101/ gr.3732505.
The most conspicuous differences between the human and chimpanzee karyotypes are the fusion that gave rise to human chromosome 2 (Fan et al. 2002), several additions of heterochromatin (van Geel et al. 2002), and nine pericentric inversions involving chromosomes 1, 4, 5, 9, 12, and 15-18 (Yunis and Prakash 1982; Nickerson and Nelson 1998; de Pontbriand et al. 2002). In previous studies, we characterized the breakpoints of the pericentric inversions of the chimpanzee chromosomes homologous to HSA 17 and HSA 18 (Kehrer-Sawatzki et al. 2002; Goidts et al. 2004). The breakpoints of the inversions of chimpanzee chromosomes homologous to HSA 4, HSA 9, and HSA 12 were regionally localized by Nickerson and Nelson (1998) and Montefalcone et al. (1999). The subsequent characterization of the breakpoints at the nucleotide level has indicated that the inversion of PTR XII (homologous to HSA 12) was associated with chimpanzee-specific sequence duplications (Kehrer-Sawatzki et al. 2005a; Shimada et al. 2005), whereas the inversion of PTR IV (equivalent to HSA 4) was mediated by inexact inverted repeats (Kehrer-Sawatzki et al. 2005b).

Here, we report the detailed study of the pericentric inversion of PTR XVI, homologous to HSA 16. By means of FISH analy- 
sis and homology searches, we identified breakpoint-spanning BAC clones from both the human and chimpanzee genomes and used them to characterize the inversion breakpoints at the molecular level. According to the banding patterns of hominoid karyotypes derived by Yunis and Prakash et al. (1982), the gorilla (GGO) manifests a pericentric inversion of its HSA 16 homolog (GGO XVI) that appears to be quite similar to the inversion of PTR XVI. We therefore investigated whether the breakpoints of the inversions of PTR XVI and GGO XVI could be identical. Our findings indicate, however, that the inversions of PTR XVI and $G G O \mathrm{XVI}$ are located at nonidentical positions and must therefore have occurred independently in the two lineages.

\section{Results}

\section{Molecular description of the inversion breakpoints}

In order to characterize the breakpoints of the inversion that distinguishes PTR XVI from HSA 16, FISH was performed with human BAC clones that map to 16p11-p12 and 16q11-q12 respectively. The results of these FISH experiments are presented in Table 1. Human BAC RP11-696P19, the proximal clone from the most centromeric contig (NT_010498) on 16q11.2, exhibited a split signal on PTR XVI (Fig. 1A,B). Although BLAST analyses of BAC RP11-696P19 indicated significant homology to scaffold_32947 of the chimpanzee draft genome sequence, a 4.8 -Mb segment from this scaffold was found to be homologous to sequences on HSA16q11.2, whereas a $1.3-\mathrm{Mb}$ segment was homologous to proximal HSA 16p (Fig. 2A,B). Moreover, the comparison between the PTR scaffold_32947, which is the most proximal scaffold on PTR XVIq, and the human chromosome 16 sequences clearly indicated the presence of an inversion and confirmed that HSA BAC RP11-696P19 spans the evolutionary breakpoint.

To identify an HSA BAC clone spanning the inversion breakpoint on the p-arm, we analyzed the $1.3-\mathrm{Mb}$ fragment of scaffold_32947 that exhibited homology to HSA 16p. The distal part of this $1.3-\mathrm{Mb}$ fragment was found to be homologous to a $144-\mathrm{kb}$ stretch of human BAC CTD-2144E22 sequence (Fig. 2B). FISH analyses confirmed that this BAC spans the inversion breakpoint, since hybridization signals were detected on both PTR XVIp and
XVIq (Fig. 1C,D). The breakpoint is located within the very distal portion of BAC CTD-2144E22; most of the corresponding sequence has been inverted and is located on PTR XVIq (Fig. 1D).

BLAST analysis with HSA BAC RP11-696P19 was then used to identify breakpoint-spanning BACs from the chimpanzee genome: RP43-007E19 and RP43-001I03. The map positions of these clones are indicated in Figure 2. FISH analyses using these PTR BACs revealed split signals on HSA 16p and 16q (Fig. 1E-H), confirming that the BACs do indeed span the inversion breakpoints. To validate the mapping of the inversion breakpoints, we performed FISH on chromosomes of the bonobo (Pan paniscus, PPA), the other chimpanzee species. Human BACs RP11-696P19 and CTD-2144E22 were also found to be breakpoint-spanning in the bonobo, whereas BAC RP11-20D16 is inverted on PPA XVIq (data not shown).

To narrow down the positions of the inversion to specific human and chimpanzee breakpoint-spanning BACs, various PCR analyses were performed. Primers were designed by reference to the sequences of the human BACs (summarized in Supplemental Table 1). In relation to the human sequence, the 16q breakpoint maps to between positions 114667-114943 of BAC RP11-696P19 whereas the $16 \mathrm{p}$ breakpoint occurred between $144-\mathrm{kb}$ and $162-\mathrm{kb}$ of BAC CTD-2144E22.

The PTR XVIq breakpoint was then characterized at the nucleotide level by comparing the sequence of the chimpanzee scaffold_32947 with that of the human BACs, RP11-696P19 and CTD-2144E22. The break in homology between HSA 16q and PTR XVIq was found to occur at position 114711 of BAC RP11-696P19 (Fig. 3), and the breakpoint on the p-arm was mapped to a short single-copy segment of 756-bp located between an L1-element and a stretch of HSATII satellites (Figs. 3, 4A). Careful analysis of the chimpanzee sequence of PTR XVIq revealed the addition of two nucleotides (AC) at the inversion junction. Under the assumption that HSA 16 represents the status of the ancestral chromosome, the breakpoint on HSA 16p occurred between two copies of an octanucleotide repeat (TGTGAAAG) whereas the breakpoint on HSA 16q occurred within one copy of a juxtaposed pentanucleotide repeat (AATGA) (Fig. 3). It can also be seen that the core of the juxtaposed pentanucleotide repeat (AAT GAAATGA) on $16 \mathrm{q}$ corresponds exactly to the core TGAAA motif of both $16 \mathrm{p}$ octanucleotide repeat sequences. When q-and p-arm

Table 1. Human BACs used as FISH probes to identify the breakpoint regions of the pericentric inversion of chimpanzee (PTR) and gorilla (GGO) chromosome XVI

\begin{tabular}{llccccc}
\hline BAC & Accession no. & Contig & $\begin{array}{c}\text { Position on HSA16 in } \\
\text { UCSC, May 2004 release }\end{array}$ & $\begin{array}{c}\text { Position } \\
\text { in HSA }\end{array}$ & $\begin{array}{c}\text { Position } \\
\text { in PTR }\end{array}$ & $\begin{array}{c}\text { Position } \\
\text { in GGO }\end{array}$ \\
\hline RP11-426C22 & AC009093.10 & NT_010393 & $28,987,481-29,195,477$ & $\mathrm{p}$ & $\mathrm{p}$ & - \\
RP11-341P6 & AC139476.3 & NT_010393 & $33,186,123-33,372,826$ & $\mathrm{p}$ & $\mathrm{p}$ & - \\
RP11-347N4 & AC141283.3 & NT_010393 & $33,556,175-33,754,470$ & $\mathrm{p}$ & $\mathrm{p}$ & $\mathrm{p}$ \\
RP11-598D12 & AC133561.4 & NT_010393 & $33,597,610-33,767,476$ & $\mathrm{p}$ & $\mathrm{p}$ & $\mathrm{p}$ \\
CTD-2522B17 & AC136932.4 & NT_010393 & $33,758,770-33,930,651$ & $\mathrm{p}$ & $\mathrm{p}$ & $\mathrm{p} / \mathrm{q}$ \\
CTD-2144E22 & AC135776.3 & NT_024773 & $34,030,652-34,199,197$ & $\mathrm{p}$ & $\mathrm{p} / \mathrm{q}$ & $\mathrm{q}$ \\
RP11-20D16 & AC092133.2 & NT_024773 & $34,067,271-34,224,164$ & $\mathrm{p}$ & $\mathrm{q}$ & $\mathrm{q}$ \\
RP11-14K3 & AC092325.2 & NT_024773 & $34,620,966-34,774,594$ & $\mathrm{p}$ & $\mathrm{q}$ & $\mathrm{q} / \mathrm{q}$ \\
RP11-65B9 & AC109490.3 & NT_024773 & $34,982,278-35,143,302$ & $\mathrm{p}$ & $\mathrm{q}$ & $\mathrm{q}$ \\
RP11-696P19 & AC106819.3 & NT_010498 & $44,943,303-45,066,095$ & $\mathrm{q}$ & $\mathrm{p} / \mathrm{q}$ & $\mathrm{q}$ \\
RP11-46D6 & AC092368.3 & NT_010498 & $45,058,799-45,229,361$ & $\mathrm{q}$ & $\mathrm{q}$ & $\mathrm{q}$ \\
RP11-93O14 & AC012186.8 & NT_010498 & $45,114,469-45,275,991$ & $\mathrm{q}$ & $\mathrm{q}$ & $\mathrm{q}$ \\
RP11-523L20 & AC007857.2 & NT_010498 & $46,457,936-46,605,357$ & $\mathrm{q}$ & $\mathrm{q}$ & $\mathrm{q}$ \\
RP11-44110 & AC026470.6 & NT_010498 & $47,019,209-47,188,774$ & $\mathrm{q}$ & $\mathrm{q}$ & \\
\hline
\end{tabular}

"- ", not performed. 

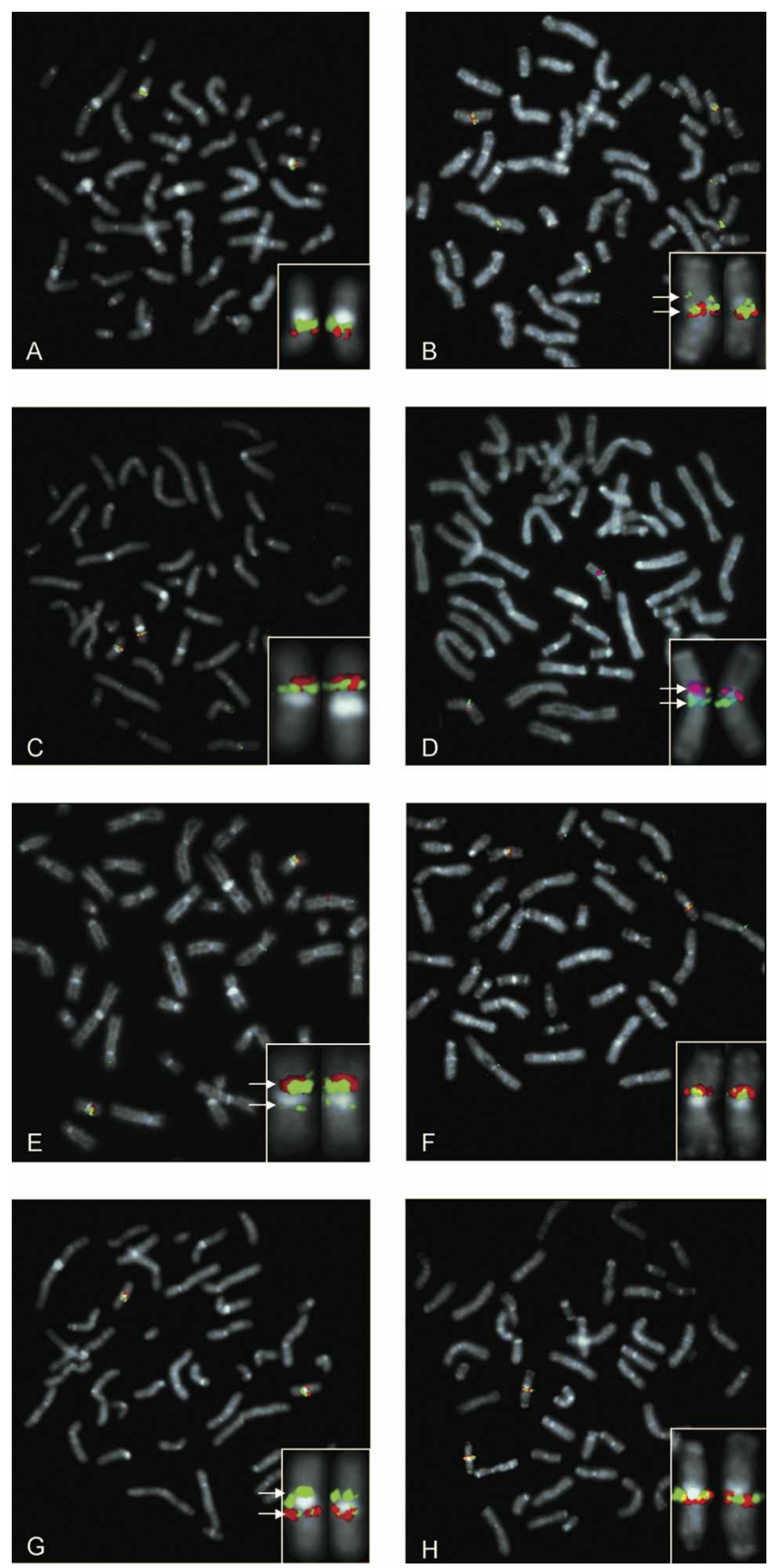

Figure 1. Comparative FISH analysis of breakpoint-spanning BACs (arrows indicate split signals). $(A, C, E, G)$ Human metaphases. $(B, D, F, H)$ Chimpanzee metaphases. $(A, B)$ FISH performed with HSA BACS RP11-696P19 (green) from 16q11.2 and RP11-44I10 (red) from 16q12.1. (C,D) HSA BACs CTD-2144E22 (green) and RP11-598D12 (red), both from HSA16p. In PTR, BAC CTD-2144E22 is split by the inversion. $(E, F)$ PTR BAC RP43$007 \mathrm{E} 19$ (green) and HSA BAC RP11-347N4 (red) from HSA 16p12. PTR BAC RP43-007E19 is breakpoint-spanning, since signals are evident at HSA $16 \mathrm{p} 11.2$ and $16 \mathrm{q} 11(E)$. On PTR XVIp, a single signal of PTR RP43007 19 is present $(F) .(G, H)$ PTR BAC RP43-001103 (green) and HSA BAC RP11-46D6 (red). PTR BAC RP43-001I03 hybridized to a single location on PTR XVIq $(H)$ but was split by the inversion on HSA $16(G)$.

breakpoint sites were further compared, a 22-base pair (bp) direct repeat was also identified. This local homology between 16p and $16 \mathrm{q}$ is indicated in Figure 3 by a wavy line.

\section{Putative mechanism underlying the inversion}

RepeatMasker analysis indicated the presence of long stretches of HSATII satellites in HSA BACs RP11-696P19 and CTD-2144E22, spanning 70-kb and 20-kb, respectively. Pairwise sequence comparisons of both BACs further indicated the presence of two inverted repeats of $\sim 23 \mathrm{~kb}$ with $97 \%$ sequence similarity between them (Fig. 4A). Given that HSA 16 represents the ancestral form of the chromosome, it appears likely that these inverted repeats, together with their flanking HSATII satellite sequences, facilitated the inversion, although the breakpoints did not occur directly within the repeats (Fig. 4B,C).

\section{Expression of the genes surrounding the breakpoints}

The inversion of PTR XVI did not interrupt the protein-coding region of a gene nor, as far as we can discern, did it disrupt the integrity of any known gene sequence. It nevertheless remains possible that the inversion could have influenced the expression of genes residing in the vicinity of the breakpoints. This notwithstanding, none of the genes immediately flanking the breakpoints had been previously identified as being differentially expressed between PTR and HSA in microarray-based expression analyses (Enard et al. 2002; Caceres et al. 2003; Karaman et al. 2003; Uddin et al. 2004). These genes are listed in Supplemental Figure 1 together with their approximate distances from the breakpoints. Since the genes LOC401848 and MGC34800, which are close to the p-arm breakpoint, and FLJ43980 mapping next to the q-arm breakpoint were not represented on the microarrays used in the aforementioned studies, we analyzed their expression by real-time PCR. Owing to its proximity to the q-arm breakpoint, we also chose to analyze the SHCBP1 gene in the same way. The expression levels of these four genes were measured in human fibroblasts and lymphocytes and then compared with the corresponding levels measured in various chimpanzee $(P T R)$, bonobo (PPA), gorilla $(G G O)$, and orangutan $(P P Y)$ cell lines. The results of these experiments are summarized in Supplemental Table 2. Most notably, the SHCBP1 gene was found to be expressed at a 100-fold higher level in two human lymphoblastoid cell lines compared to their gorilla counterparts. Further analyses, employing RNA isolated from primary tissues of several gorillas, would however be required to confirm this expression difference.

\section{Evolutionary history of the breakpoint regions}

FISH analysis revealed that HSA BAC RP11-696P19 hybridized not only to HSA 16, but also to HSA 1 . To investigate the evolutionary history of the sequences represented by this BAC, we performed FISH analyses on chromosomes of other primates. RP11-696P19 signals were detected on chromosomes homologous to HSA 16 and HSA 1 in chimpanzee, bonobo, and gorilla (Fig. 5B). Interestingly, with metaphases from orangutan (PPY) and macaque (MFA), BAC RP11-696P19 did not hybridize to the HSA 16 homologs, but rather hybridized exclusively to the equivalents of $H S A 1$. The MFA I and PPY I chromosomes may be distinguished by a pericentric inversion which occurred in the Old World monkey lineage about 25 million years ago (Mya), after their divergence from the hominoid lineage (Dutrillaux et al. 1979; Maresco et al. 1998). After the separation of the orangutan lineage from that of the other great apes, a duplicational interchromosomal transposition to the ancestral chromosome XVIq occurred, which involved the region covered by BAC RP11696P19. This was followed by the pericentric inversion on PTR

\section{Genome Research}

www.genome.org 
A

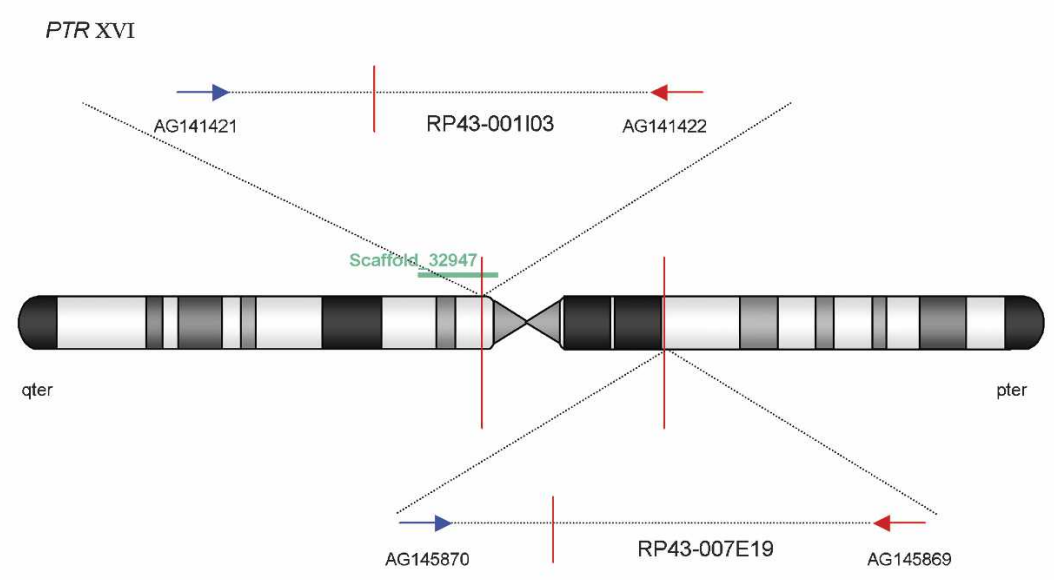

B

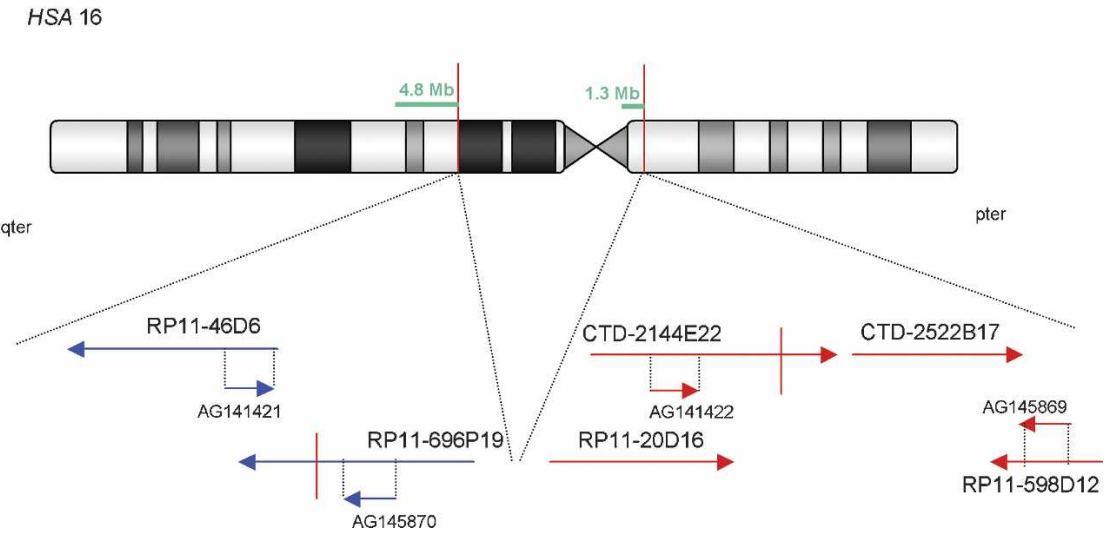

Figure 2. Ideogram of human chromosome 16 (HSA 16; B) and its chimpanzee homolog (PTR XVI; $A)$. BACs used for FISH analyses are indicated in blue (HSA 16q) and red (HSA 16p). Inversion breakpoints are denoted by vertical red lines. The green lines represent the chimpanzee scaffold_32947, which is split on human chromosome 16.

XVI. The region corresponding to BAC RP11-696P19 was also split by a pericentric inversion on GGO XVI, homologous to $H S A$ 16 (Fig. 5A).

Human BAC CTD-2144E22 hybridized to the p-arm of the HSA 16 homologs of both macaque and orangutan. However, on PTR XVI, PPA XVI, and GGO XVI, this BAC displayed a split signal on the p- and q-arms (data not shown). Taken on their own, these findings are not inconsistent with the view that gorilla and chimpanzee share the same pericentric inversion.

\section{Breakpoint position of the GGO XVI inversion}

FISH experiments with the human BACs used to narrow down the inversion breakpoint regions in PTR XVI yielded hybridization patterns in the gorilla that were comparable to those observed in the chimpanzee (summarized in Table 1). This indicates that gorilla does indeed have an inversion, and that the breakpoints are either identical or at least reside in the same genomic region as in the common chimpanzee.

To determine whether or not the inversion breakpoints of PTR XVI and GGO XVI occurred at identical sites, FISH was performed with PTR BACs that span the inversion breakpoints on PTR XVI. These chimpanzee BACs, RP43-007E19 and RP43001I03, yielded split signals on GGO XVI (data not shown). This finding implies that it is extremely unlikely that the PTR and GGO inversion breakpoints are identical in terms of their location. As previously mentioned, human BACs CTD-2144E22 and RP11696P19 are split by the inversion in both PTR XVI and GGO XVI. It may therefore be concluded that although the inversion breakpoints in PTR XVI and GGO $\mathrm{XVI}$ are in close proximity to each other, they are nevertheless nonidentical.

To investigate these breakpoints at the nucleotide level, we performed breakpoint-spanning PCR with primer pair P2 (listed in Supplemental Table 1). The primers were designed according to the sequence of HSA BAC RP11-696P19 and flank the inversion breakpoint on HSA 16q (Fig. 6). PCR P2 was negative in $P T R$, but positive in $H S A$ and $G G O$ (data not shown). Thus, the sequence at the q-arm breakpoint must differ in $G G O$ XVI from that in PTR XVI. By screening a gorilla BAC library with PCR probes (listed in Supplemental Table 3), we identified breakpoint-spanning BACs CH255-551C4 (AY822675) and CH25539D12 (AY822676, AY822677) from the gorilla genome. The positions of these $G G O$ BAC clones with respect to the breakpoints were confirmed by PCR analysis (P1-P10) (schematically indicated in Fig. 6) and by sequencing the BAC ends. GGO BAC CH255-39D12 mapped distal to the inversion breakpoint in GGO XVIq and was positive for PCR markers P9, P3, P1, and P8 (Fig. 6). This served to confirm the interspecies heterogeneity of the q-arm breakpoints in PTR XVI and GGO XVI. GGO BAC CH255-551C4 was positive for PCR markers P6, P7, P10 and mapped to GGO XVIp. Since PCR marker P6 is directly flanked by HSATII repetitive sequences, further localization of the gorilla p-arm breakpoint by PCR was not possible. We nevertheless conclude that the inversion breakpoint on $G G O$ XVIp lies close to or within the HSATII satellites, as found for the breakpoint on PTR XVIp. It is likely that the GGO XVI inversion was mediated by intrachromosomal recombination within HSATII, as schematically drawn in Figure 7.

\section{Discussion}

The breakpoints of the pericentric inversion of PTR XVI, homologous to HSA 16, were characterized by FISH and comparative sequence analyses of breakpoint-spanning BAC clones from the human and chimpanzee genomes. The breakpoints occurred within sequences homologous to human HSA BACs RP11-696P19 (16q11.2) and CTD-2144E22 (16p11.1-p11.2) in single-copy sequences close to HSATII satellites, but do not appear to have disrupted any gene coding regions (Figs. 1-3). HSA BACs RP11696P19 and CTD-2144E22 also spanned the inversion breakpoints of the chromosome 16 homolog in the bonobo, the other chimpanzee species, which became separated from the common 


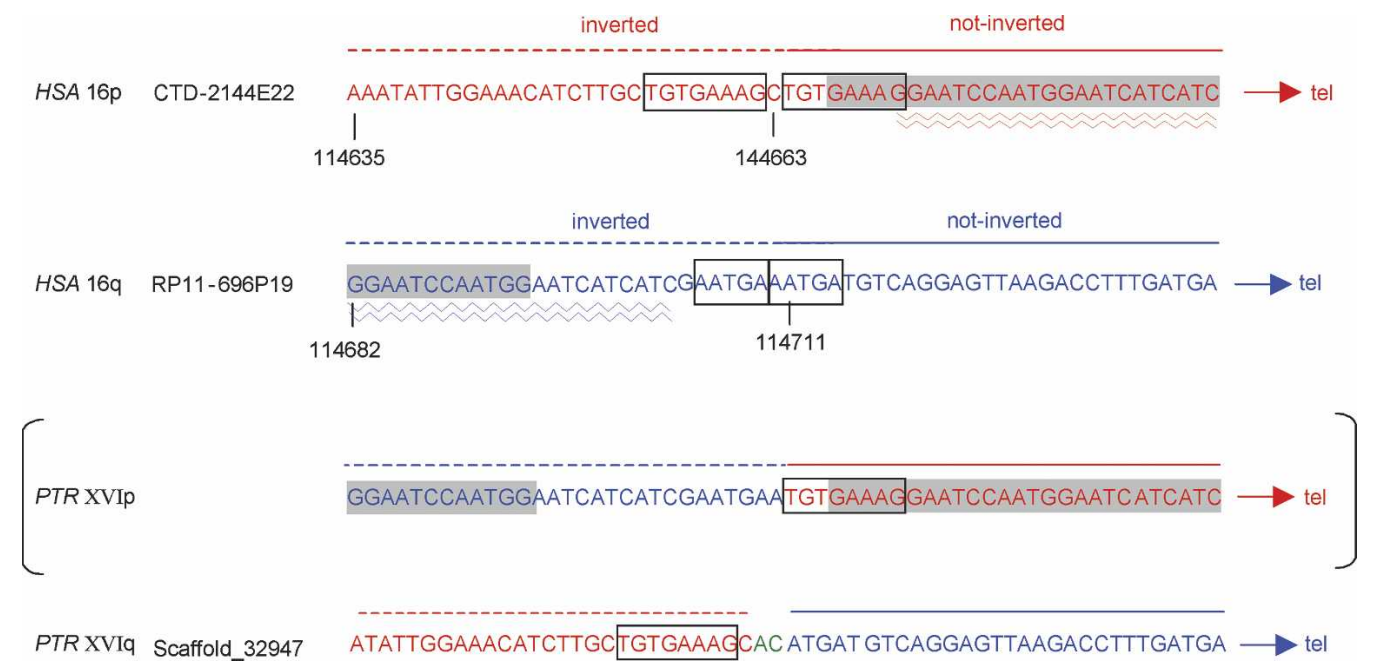

Figure 3. Positions of the breakpoints at the nucleotide level. The sequence in blue represents a portion of HSA BAC RP11-696P19 (16q). The sequence in red is derived from BAC CTD-2144E22 (HSA 16p). The boxes indicate the relative positions of the octanucleotide repeat that flanks the HSA16p breakpoint and the pentanucleotide repeat at 16q. A 22-bp direct repeat found at the breakpoints on chromosomes $16 \mathrm{p}$ and $16 \mathrm{q}$ is underlined by wavy lines. The HSATII satellite sequences are underlined in gray. The analysis of the chimpanzee sequence in PTR XVIq revealed the presence of two additional nucleotides (AC, in green). Parentheses serve to indicate that the breakpoint region at PTR XVIp, within the HSATIl satellite DNA, was inferred from the human sequence, and was not sequenced in PTR owing to the abundance of repetitive satellite sequences.

chimpanzee, Pan troglodytes, 0.86-2 Mya (Yoder and Yang 2000; Won and Hey 2005). Intriguingly, the inversion of PTR XVI was not observed by Martin et al. (2004) in their comparison of the HSA 16 sequence with homologous sequences available in other vertebrates, including the chimpanzee. One possible reason for this might be that the scaffolds of the draft chimpanzee genome sequence from the breakpoint regions had not been assembled into larger contigs and, in the absence of FISH data, this could have hampered the assignment of the orientation of these scaffolds.

Comparative FISH analysis in other primates revealed the evolutionary history of the q-arm breakpoint region (Fig. 5). This region, represented by HSA BAC RP11-696P19, is derived from an ancestral primate chromosome homologous to HSA 1. After the separation of the orangutan from the common ancestral lineage shared with the African hominoids, a duplication of the region in question occurred followed by the interchromosomal transposition of the duplicated copy to the ancestral chromosome 16q. The most parsimonious explanation for our findings is that among the hominoids, humans retained the ancestral form of chromosome 16, whereas GGO and PTR acquired pericentric inversions of their respective homologs. The inversions of $G G O \mathrm{XVI}$ and PTR XVI were not detected during the molecular cytogenetic studies previously performed by Misceo et al. (2003) because the probes that they used are located distally to the respective breakpoints.

Comparative analysis of the inversion breakpoint regions in GGO XVI and PTR XVI indicated that whereas the p-arm breakpoints were located within or very close to HSATII satellite DNA, the q-arm breakpoints occurred at nonidentical locations (Fig. 6). Owing to the repetitive nature of the HSATII satellites, it was not possible to compare the p-arm breakpoints in GGO XVI and PTR XVI. This notwithstanding, q-arm breakpoint heterogeneity is supportive of the view that the pericentric inversions of GGO XVI and PTR XVI occurred independently in the two lineages. PCR with primer pair P2, designed according to the sequence of $H S A$ BAC RP11-696P19 and flanking the inversion breakpoint on HSA 16q, was negative in PTR, but positive in $H S A$ and GGO (data not shown). Sequence analysis of the PCR products also indicated an identical sequence in HSA and GGO at the site where the inversion/fusion took place in $P T R$. The sequence amplified by primer pair P2 has not been deleted in PTR, but rather was split by the inversion (Figs. 3, 6). This provides unequivocal confirmation that the inversions of GGO XVI and PTR XVI occurred at different sites.

Although pericentromeric regions are generally enriched for duplicated sequences, considerable variability with respect to the length of paralogous sequences is apparent. The 16p11-12 region belongs to the category of region with extensive zones of duplication ranging from 500-kb to 5.5-Mb in length (She et al. 2004). Detailed analyses of HSA $16 \mathrm{p} 11$ have identified multiple paralogous sequences, including sequences derived from portions of the $S L C 6 A 8, H E R C 2, A L D$, and variable heavy chain immunoglobulin genes (Eichler et al. 1996, 1997; Ji et al. 2000a, 2000b; Horvath et al. 2001). The recent amplification and transposition of a duplicated segment of $\sim 20 \mathrm{~kb}$ throughout a $15-\mathrm{Mb}$ region of human chromosome $16 \mathrm{p}$ has also been described (Johnson et al. 2001). The dispersal of this $20-\mathrm{kb}$ segment has been accompanied by considerable variation in chromosomal map location and copy number among hominoid species. In humans, the NPIP gene family (nuclear pore complex interacting protein, also termed morpheus) has been shown to reside within these duplicated segments. Sequence comparisons have revealed that members of this gene family have been under a considerable degree of positive selection, especially in the human lineage (Johnson et al. 2001). The most proximal of these $16 \mathrm{p}$ duplications, termed LCR16a, maps $\sim 5-\mathrm{Mb}$ distal to the inversion breakpoint. According to the Ensembl map of the corresponding region, amplified copies of the TP53TG3 gene (see Supplemental Fig. 1) are also present in the vicinity of the p-arm breakpoint. BLAST analyses of the distal part of BAC RP11-696P19, which spans the 16q breakpoint, were indicative of a 26-kb duplicon termed DC3609 according to the human genome segmental duplication database. Paralogs of DC3609 are dispersed within a $\sim 3-\mathrm{Mb}$ region

\section{Genome Research}

www.genome.org 


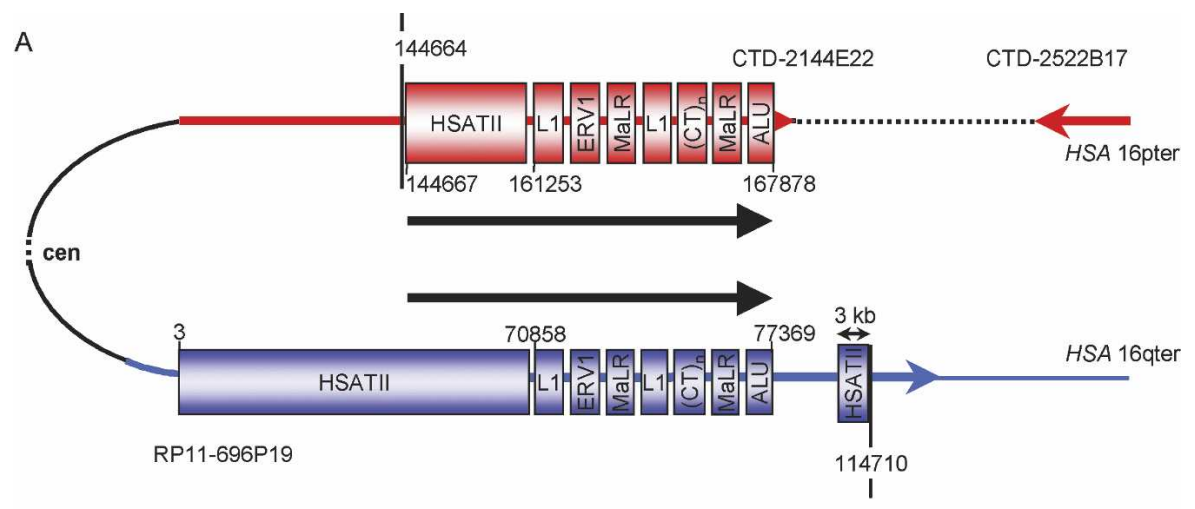

B

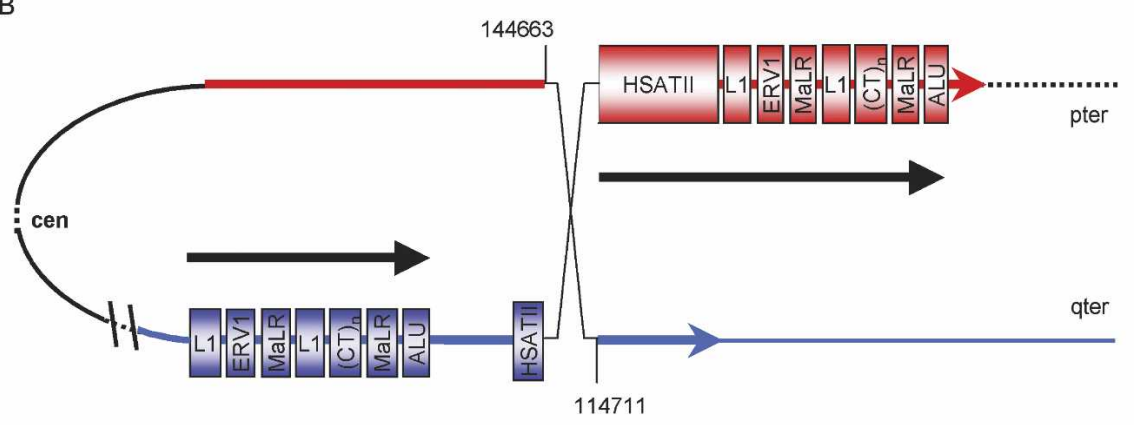

C

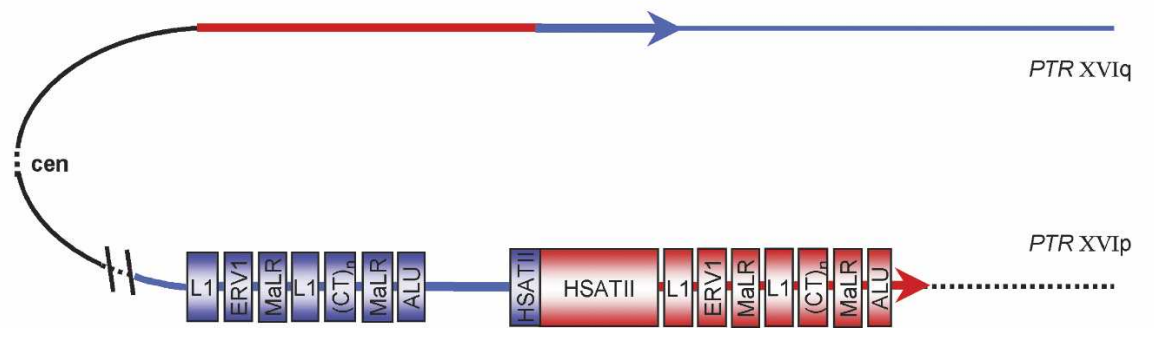

Figure 4. Hypothetical model to explain the pericentric inversion of PTR XVI homologous to HSA 16. (A) Breakpoint-spanning HSA BACs RP11-696P19 and CTD-2144E22 are indicated by blue and red lines, respectively. The black arrows denote the $23-\mathrm{kb}$ inverted repeat comprising repetitive elements and HSATII satellite DNA. The high degree of similarity conferred by the inverted repeats may have brought the chromosomal regions $16 \mathrm{p} 11-\mathrm{p} 12$ and $16 \mathrm{q} 11-\mathrm{q} 12$ together, thereby mediating an intrachromosomal recombination event $(B)$. The numbers indicate the nucleotide positions according to the numbering of BAC clones RP11-696P19 and CTD-2144E22. (C) Schematic of the rearrangement on PTR XVI resulting from the recombination between the $\mathrm{p}$ - and $\mathrm{q}$-arms. cen, centromere.

distal to the p-arm breakpoint. BLAST analyses of HSA BAC CTD2144E22, spanning the p-arm breakpoint, revealed a 20-kb duplicon (DC3606) in the proximal part of the BAC. Paralogous sequences of DC3606 are located in 16q but at least $200 \mathrm{~kb}$ distal to the q-arm breakpoint.

It has been suggested that the abundance of duplicated sequences in pericentromeric and subtelomeric portions of mammalian chromosomes gives rise to genomic instability via unequal crossing over during meiosis and may thus serve to drive evolution (Eichler 1998; Samonte and Eichler 2002). The high concentration of paralogous segments within the pericentromeric region of chromosome 16 and its evident plasticity are reflections of its likely importance in an evolutionary context. Further evidence for the instability of proximal $16 \mathrm{p}$ and $16 \mathrm{q}$ comes from reports of pathological breakpoints in these regions. Thus, the 16p11 and 16q11-12 regions are known to be involved in recurring rearrangements in acute myeloid leukemia (Panagopoulos et al. 1994; Sharma et al. 1999; Yamamoto et al. 2001), Ewing's tumor (Shing et al. 2002), lymphoma (Ueda et al. 2002), and liposarcoma (Willeke et al. 1998).

About $5 \%$ of our genome is composed of segmental duplications or lowcopy repeats. In several instances, duplications have been found at, or in close proximity to, evolutionary breakpoints. It has been suggested that duplicated segments could have played a pivotal role during genome evolution in higher primates (Samonte and Eichler 2002; Shaw and Lupski 2004). For example, the telomeric fusion of two acrocentric chromosomes that gave rise to HSA 2 appears to have been mediated by a duplication-driven mechanism (Fan et al. 2002). Similarly, the breakpoints associated with the gorilla-specific translocation $t(4 ; 19)$ occurred within a low-copy repeat (LCR)-rich region and has been shown to be associated with a gorillaspecific duplication of $\sim 250 \mathrm{~kb}$ (Stankiewicz et al. 2001, 2004). In addition, the breakpoints of the pericentric inversion that distinguishes HSA 15 from its orthologous PTR counterpart map to segmental duplication clusters (Locke et al. 2003), whereas the inversion of the chimpanzee chromosome (PTR XVIII) homologous to HSA 18 was probably mediated by a $20-\mathrm{kb}$ duplicated segment (Dennehey et al. 2004; Goidts et al. 2004). In general, it has been noted that primate-specific segmental duplications are overrepresented at the breakpoints of syntenic blocks between the human and mouse genomes (Armengol et al. 2003; Bailey et al. 2004).

Human chromosome 16 is known to manifest one of the highest levels of segmentally duplicated sequences among the autosomes (Martin et al. 2004). Approximately $10 \%$ of chromosome 16 consists of segmental duplications, and nearly $9 \%$ of genome-wide human duplication alignments map to this chromosome. It should also be noted that as much as $7 \%$ of the mass of human chromosome 16 has been added by segmental duplication events occurring within the last 10 million years (Myr) (Martin et al. 2004). In addition, the $16 \mathrm{p} 11$ pericentromeric region harbors a high concentration of interchromosomal duplications, accounting for $55 \%$ of all chromosome 16 interchromosomal alignments (Martin et al. 2004). Finally, large tracts of interstitial alpha-satellite DNA have been identified within proximal 16p11, and it is possible that such sequences have played an important role in the frequent evolutionary interchromosomal exchange of pericentromeric DNA (Martin et al. 2004).

Detailed analyses of pericentromeric regions have revealed that paralogous sequences are often located in close proximity to 


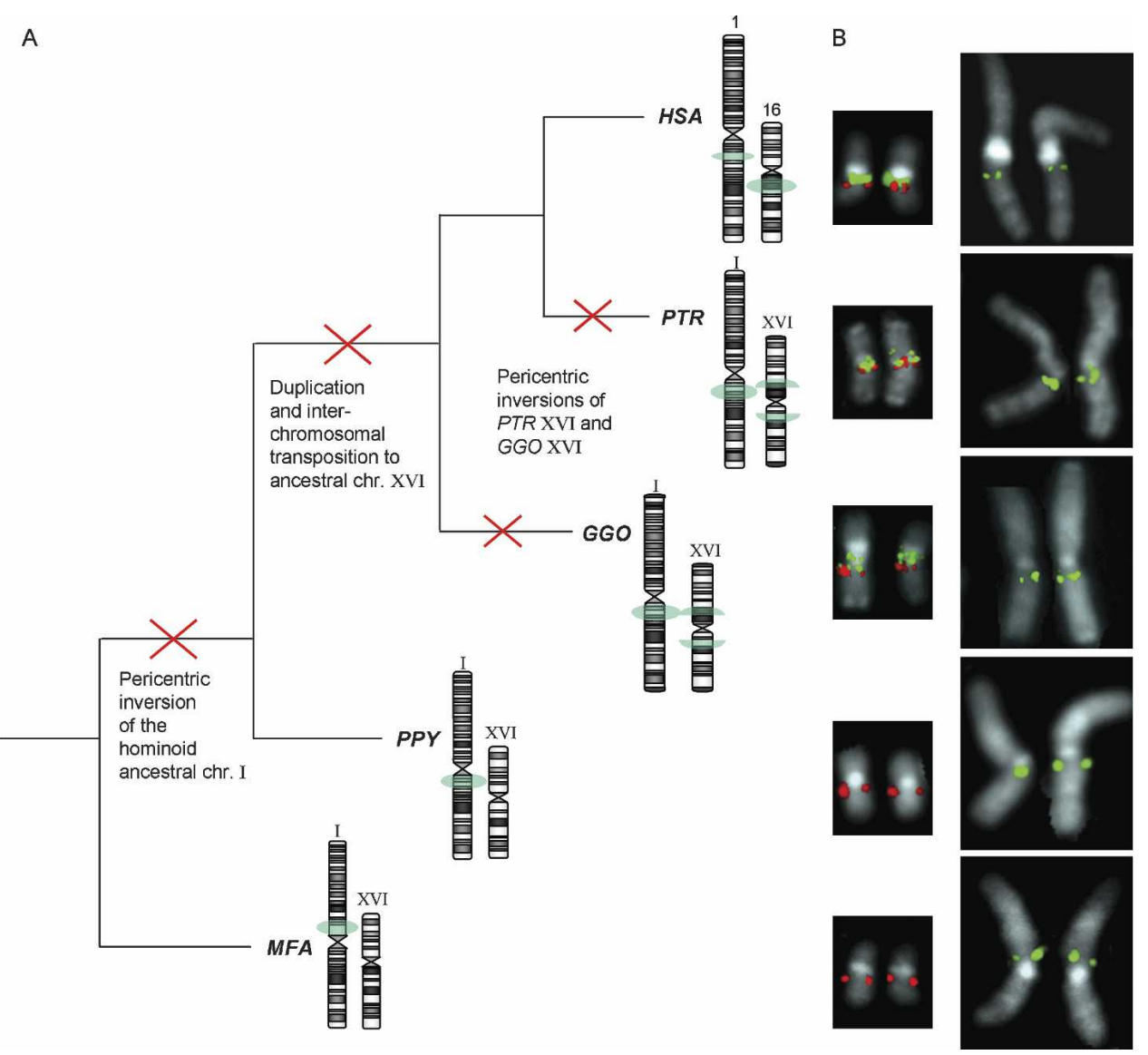

Figure 5. FISH analyses designed to follow the evolutionary history of the region represented by HSA BAC RP11-696P19. (A) Ideogram of the chromosome I and chromosome XVI homologs in Macaca fascicularis (MFA), Pongo pygmaeus (PPY), Gorilla gorilla (GGO), Pan troglogdytes (PTR), and Homo sapiens (HSA). The positions of the regions homologous to HSA BAC RP11-696P19, as determined by FISH, are schematically indicated in green. The red crosses indicate the occurrence of the respective rearrangements (B) FISH hybridization pattern of human BACs RP11-696P19 (green) and RP11-44I10 (red) from 16q11-12 on chromosome 16 (left) and 1 (right) homologs. In metaphase spreads of MFA and PPY, signals from RP11-696P19 were found exclusively on chromosome I.

satellite sequences (Horvath et al. 2000, 2001; Jackson 2003). In the case of chromosome $16 \mathrm{p}$, paralogous sequences extend over several megabases in the centromeric region (Martin et al. 2004). Our findings are consistent with the pericentric inversions of PTR XVI and GGO XVI having been mediated by recombination between paralogous sequence blocks and stretches of HSATII satellite. An inverted repeat of 23-kb, comprising satellites, LINE elements, Alu sequences, and other repetitive elements was identified in the breakpoint regions in 16p11-12 and 16q11-12 (Figs. 4, $7)$. It is likely that the high degree of sequence similarity manifested by these inverted repeats served to mediate the formation of the intrachromosomal inversion loop, thereby facilitating the respective regions coming into close proximity with each other. The various local sequence homologies apparent within and between the inversion breakpoints [viz. the octanucleotide repeat (TGTGAAAG) at the $16 \mathrm{p}$ breakpoint, the pentanucleotide repeat (AATGA) at the $16 \mathrm{q}$ breakpoint, the TGAAA motif common to the $16 \mathrm{p}$ and $16 \mathrm{q}$ breakpoints, and the 22 -bp direct repeat that flanks the $16 \mathrm{p}$ and $16 \mathrm{q}$ breakpoints (Fig. 3)] could then account for the inversion occurring at the sites that it did. Direct repeats are known to form "slipped structures," and these non-B DNA conformations could have contributed to the formation of local breaks that then led to the inversion in PTR. Non-B DNA struc- tures have been shown to play a role in numerous genomic rearrangements associated with human disease (for review, see Bacolla and Wells 2004).

Breakpoint comparisons by both FISH and PCR analysis strongly imply that the inversions of PTR XVI and GGO XVI are independent events (Fig. 6, Table 1). Indeed, the q-arm breakpoint in $G G O$ XVI appears to be at least $70 \mathrm{~kb}$ distant from the breakpoint in PTR XVIq. With respect to the $\mathrm{p}$-arm breakpoints of the two inversions, breakpoint identity can neither be confirmed nor excluded, since the breakpoints were mapped to highly repetitive HSATII DNA.

The gorilla branched off 6.2-8 Mya from the main hominoid lineage; the chimpanzee separated later, some 4.6-6.2 Mya (Chen and Li 2001). Had the GGO and PTR inversions been identical by descent, then either humans would have possessed this inversion as well, or the human chromosome would have been the derived one.

Although the GGO XVI and PTR XVI inversions appear to have been independent events, the breakpoints nevertheless occurred in the same chromosomal regions. Although this finding is, as far as we are aware, unprecedented during vertebrate evolution, multiple independent yet cytologically identical inversions have been demonstrated by DNA sequence analysis in the

\section{Genome Research}

www.genome.org 

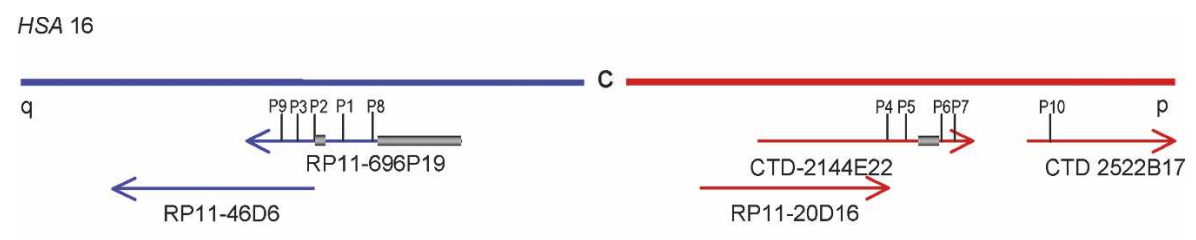

PTR XVI

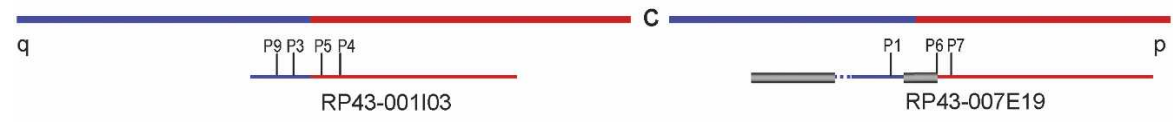

GGO XVI

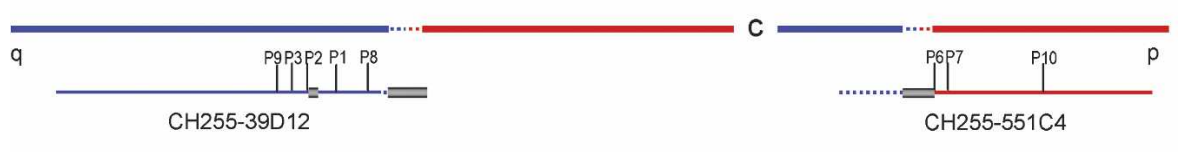

HSATII

Figure 6. Scheme indicating the breakpoint regions of the inversions on PTR XVI and GGO XVI in comparison with HSA 16. The positions of the primer pairs (P1-P10) used to compare the regions in these species are indicated. The dotted lines indicate the regions where the breakpoints of the GGO $\mathrm{XVI}$-specific inversion were assigned. HSATII satellites are represented as gray bars. Sequences from the $\mathrm{q}$-arm are indicated in blue and $\mathrm{p}$-arm sequences in red.

Anopheles gambiae complex (Caccone et al. 1998). It might be argued that the parallel evolution of the cytologically identical inversion could have been selectively favored. Indeed, since chromosome 16 is very rich in duplicated sequences, the probability that inversions with breakpoints in the same regions occur twice quite independently is likely to be rather low. However, despite the fact that HSA 16 contains the highest level of duplications among the autosomes, these duplicated regions tend to be clustered, particularly along the p-arm. In contrast to $16 \mathrm{p} 11$ 12 , the $16 \mathrm{q} 11-12$ region is not exceptionally rich in duplications. Since the pericentric inversions of both PTR XVI and GGO XVI appear to have been mediated by recombination between paralogous sequence blocks and stretches of HSATII satellite found in $16 \mathrm{p} 11$ and 16q11, these regions may have specifically facilitated inversions.

Bailey et al. (2004) have put forward a nonrandom model of chromosomal evolution that proposes that specific regions in mammalian genomes are predisposed to both recurrent smallscale duplication and large-scale evolutionary rearrangements. The occurrence of two independent inversions in GGO and PTR with breakpoints in the same regions provides clear support for this nonrandom model.

\section{Methods}

\section{Cell lines and BAC clones}

The Pan troglodytes lymphoblastoid cell line PTR-EB176 (ECACC No. 89072704), the Pan troglodytes primary fibroblast culture CP132 (PTR, ECACC No. 91012416), the orangutan (Pongo pygmaeus) lymphoblastoid cell line PPY-EB185 (ECACC No.
89072705), and the lymphoblastoid cell line EB(JC) (ECACC No. 89072703) from Gorilla gorilla were purchased from the European Collection of Cell Cultures (www.ecacc.org.uk). The Pan troglodytes lymphoblastoid cell line PTR-L2008 was a generous gift from Dr. W. Schempp (University of Freiburg, Germany). The lymphoblast cell line GM03446 from Macaca fascicularis (crab-eating macaque) was obtained from the Coriell Cell Repository. The bonobo (Pan paniscus) lymphoblastoid cell line used in these studies was established from peripheral blood samples (taken from a female animal from Frankfurt Zoo, Germany), a generous gift from Dr. Ulrich Zechner (University of Mainz, Germany). For expression analysis, two foreskin fibroblast cell lines and two EBVtransformed lymphoblastoid cell lines from human donors were used.

Fluorescence in situ hybridization (FISH)

Metaphase chromosomes were prepared from the PTR-EB176 lymphoblastoid cell line. BACs CTD-2144E22 (AC135776) and CTD-2522B17 (AC136932) were obtained from Invitrogen (www.invitrogen .com), and the other BAC clones were obtained from the BACPAC Resource Center (www.chori.org/bacpac). BAC DNA was isolated using the QIAGEN-Midi-Kit (Qiagen). At least 1 $\mu \mathrm{g}$ BAC-DNA was labeled with biotin-16-dUTP (RocheDiagnostics) and detected with FITC-avidin and biotinylated anti-avidin (Vector). In cohybridizations using two differentially labeled BACs, the second DNA sample was labeled with digoxygenin-11-dUTP (Roche-Diagnostics) and detected by mouse antidigoxygenin. A second step employed anti-mouse antibodies from rabbit coupled with Texas-Red. In a third round of detection, anti-rabbit antibodies conjugated with Texas-Red were used (Dianova). Finally, slides were counterstained with diamidinophenylindole (DAPI) and mounted with Vectashield antifade solution (Vector).

\section{Sequence alignments and database analysis}

Alignments of BAC sequences were performed by BLAST searches against genome databases (www.ncbi.nlm.nih.gov/blast/) and FASTA analyses using the Wisconsin Package, version 10.2 (Genetics Computer Group). Breakpoint regions were analyzed by reference to the Ensembl database (NCBI Build 1.1, November 2003, http://www.ncbi.nlm.nih.gov/) and the chimpanzee draft sequence (www.ensembl.org/). The search for human-specific duplications was performed using the Human Genome Segmental Duplication Database (http://projects.tcag.ca/humandup) based on the May 2004 Human Genome Assembly (hg17).

\section{BAC library screening}

In order to identify BACs from the gorilla genome that map to the breakpoint regions, colony-filter hybridizations of the CHORI-255 library (Gorilla gorilla gorilla) were performed with 40-bp synthetic probes included in the PCR products P8, P9, and P10. PCR primers (listed in Supplemental Table 3) were designed according to the sequences of human BACs. Radiolabeled 40-bp 
A

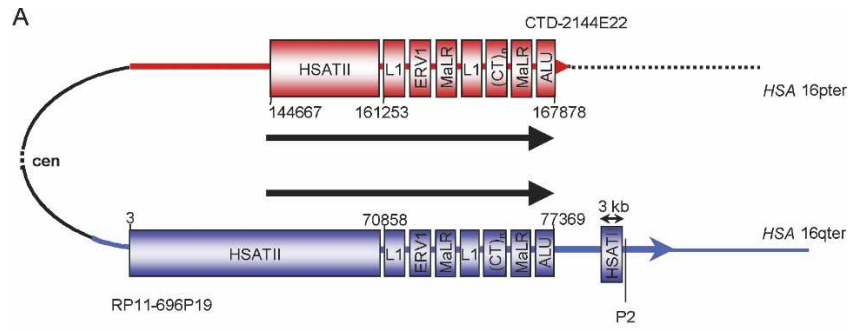

B

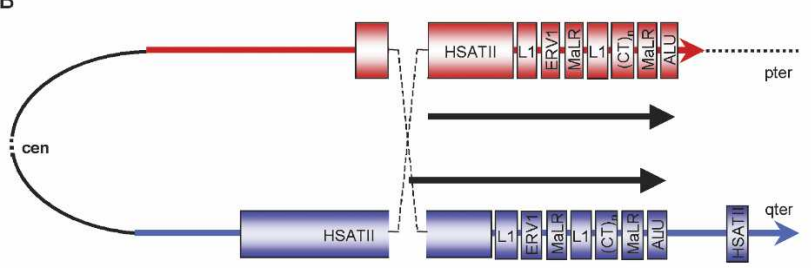

C

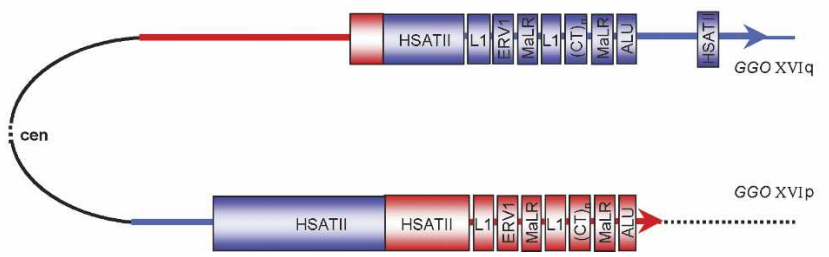

Figure 7. Model to explain the pericentric inversion of the gorilla chromosome (GGO XVI) homologous to HSA 16. P2 indicates the position of the inversion breakpoint on PTR XVIq. The numbers indicate the nucleotide positions of BAC clones RP11-696P19 and CTD-2144E22. (A) Breakpoint-spanning HSA BACs RP11-696P19 and CTD-2144E22 are indicated by blue and red lines, respectively. The black arrows denote the $23-\mathrm{kb}$ inverted repeats at the breakpoints, which are postulated to have mediated intrachromosomal recombination underlying the inversion $(B)$. The result of this recombination is the inversion on GGO XVI as shown in (C). cen, centromere.

overgo probes were created from 3'-overlapping 24-mers by extending the primers with Klenow DNA polymerase using $\mathrm{P}^{32}$ dCTP as described by Ross et al. (1999). Hybridization-positive BAC clones were first confirmed by PCR analysis with primer pair P8, and three BAC clones were shown to be true positives. Further PCR reactions were performed with primer pairs P9 and P10, thereby potentiating the identification of the breakpointspanning gorilla BACs CH255-39D12 and CH255-551C4.

\section{RNA isolation and cDNA preparation}

Cytoplasmic RNA was isolated from lymphoblastoid and fibroblast cell cultures derived from three chimpanzees and four humans, as well as lymphoblastoid cells from one bonobo, one gorilla, one orangutan, and one macaque, using the RNeasy kit (Qiagen). First strand cDNA was prepared with $2 \mu \mathrm{g}$ total RNA (as determined by absorbance), random hexamers, and the SuperScript Preamplification System (Invitrogen).

\section{Gene expression analysis}

Real-time RT-PCR was performed using the DNA-binding dye SYBR Green on a Lightcycler with the Quantitect SYBR Green
PCR kit (Qiagen). Relative quantification was used to normalize the target genes (LOC401848, FLJ43980, and SHCBP1) to an internal standard (HPRT, hypoxanthine-guanine phosphoribosyltransferase). To determine mRNA expression, a standard curve for each of the two fragments was generated. The change in gene expression was then given as the ratio of the target gene to HPRT expression. Gene-specific primers used for this analysis were 5'GCGGATCCAGAAGGACATAA3' and 5'GTTGCCCTCGATTT CAATGT3' for LOC401848, 5'TGCAAGAGAAACGTGAAA CA3' and 5'CTGCTATCTCCTGCCTTGCT3' for FLJ43980, 5'TTGGAGGAGTTGGGATGAAG3' and 5'TCCTGCTCGGAAT CAGAGTT3' for SHCBP1, three different pairs of primers for the gene MGC34800, 5'TCACCACTAAGGGCAACCTC3' and 5'ggtc gccattcattatttgg3', 5'GGATAGGAGTCGGGAAGAGC3' and 5'AC AGCTCATGCAGCAGATCC3', 5'CTTTCAGCCTGCAGTTCC TC3' and 5'ATTCCACATGTGAGTGCCAA3', and 5'gctggtgaaaa ggacctct3' and 5'cacaggactagaacacctgc $3^{\prime}$ for HPRT.

For each analysis, two Pan troglodytes lymphoblastoid cell lines were used, together with one Pan troglodytes primary fibroblast culture, and single lymphoblastoid cell lines from Pan paniscus, Gorilla gorilla, Pongo pygmaeus, and Macaca fascicularis. Two foreskin fibroblast cell lines and two EBV-transformed lymphoblastoid cell lines from human donors were also used. Each comparison was performed in triplicate.

\section{Acknowledgments}

We thank Antje Kollak and Helene Spöri for technical assistance. This research was funded by the Deutsche Forschungsgemeinschaft (DFG KE 724/2-1).

\section{References}

Armengol, L., Pujana, M.A., Cheung, J., Scherer, S.W., and Estivill, X. 2003. Enrichment of segmental duplications in regions of breaks of synteny between the human and mouse genomes suggest their involvement in evolutionary rearrangements. Hum. Mol. Genet. 12: 2201-2208.

Bacolla, A. and Wells, R.D. 2004. Non-B DNA conformations, genomic rearrangements, and human disease. J. Biol. Chem. 279: 47411-47414.

Bailey, J.A., Baertsch, R., Kent, W.J., Haussler, D., and Eichler, E.E. 2004. Hotspots of mammalian chromosomal evolution. Genome Res. 5: R23.

Cáceres, M., Lachuer, J., Zapala, M.A., Redmond, J.C., Kudo, L., Geschwind, D.H., Lockhart, D.J., Preuss, T.M., and Barlow, C. 2003. Elevated gene expression levels distinguish human from non-human primate brains. Proc. Natl. Acad. Sci. 100: 13030-13035.

Caccone, A., Min, G.S., and Powell, J.R. 1998. Multiple origins of cytologically identical chromosome inversions in the Anopheles gambiae complex. Genetics 150: 807-814.

Chen, F.C. and Li, W.H. 2001. Genomic divergences between humans and other hominoids and the effective population size of the common ancestor of humans and chimpanzees. Am. J. Hum. Genet. 68: $444-456$.

Dennehey, B.K., Gutches, D.G., McConkey, E.H., and Krauter, K.S. 2004. Inversion, duplication, and changes in gene context are associated with human chromosome 18 evolution. Genomics 83: 493-501.

de Pontbriand, A., Wang, X.P., Cavaloc, Y., Mattei, M.G., and Galibert, F. 2002. Synteny comparison between apes and human using fine-mapping of the genome. Genomics 80: 395-401.

Dutrillaux, B., Biemont, M.C., Viegas-Pequinot, E., and Laurent, C. 1979. Comparison of the karyotypes of four Cercopithecoidae: Papio papio, P. anubis, Macaca mulatta, and M. fascicularis. Cytogenet. Cell Genet. 23: 77-83.

Eichler, E.E. 1998. Masquerading repeats: Paralogous pitfalls of the human genome. Genome Res. 8: 758-762.

Eichler, E.E., Lu, F., Shen, Y., Antonacci, R., Jurecic, V., Doggett, N.A., Moyzis, R.K., Baldini, A., Gibbs, R.A., and Nelson, D.L. 1996. Duplication of a gene-rich cluster between $16 \mathrm{p} 11.1$ and Xq28: A novel pericentromeric-directed mechanism for paralogous

\section{Genome Research}

www.genome.org 
genome evolution. Hum. Mol. Genet. 5: 899-912.

Eichler, E.E., Budarf, M.L., Rocchi, M., Deaven, L.L., Doggett, N.A., Baldini, A., Nelson, D.L., and Mohrenweiser, H.W. 1997.

Interchromosomal duplications of the adrenoleukodystrophy locus: A phenomenon of pericentromeric plasticity. Hum. Mol. Genet. 6: $991-1002$.

Enard, W., Khaitovich, P., Klose, J., Zöllner, S., Heissig, F., Giavalisco, P., Nieselt-Struwe, K., Muchmore, E., Varki, A., Ravid, R., et al. 2002. Intra- and interspecific variation in primate gene expression patterns. Science 296: 340-343.

Fan, Y., Linardopoulou, E., Friedman, C., Williams, E., and Trask, B.J. 2002. Genomic structure and evolution of the ancestral chromosome fusion site in 2q13-2q14.1 and paralogous regions on other human chromosomes. Genome Res. 12: 1651-1662.

Goidts, V., Szamalek, J.M., Hameister, H., and Kehrer-Sawatzki, H. 2004. Segmental duplication associated with the human-specific inversion of chromosome 18: Further example of the impact of segmental duplications on karyotype and genome evolution in primates. Hum Genet. 115: 116-122.

Hey, J. 2003. Speciation and inversions: Chimps and humans. Bioessays 25: $825-828$

Horvath, J.E., Viggiano, L., Loftus, B.J., Adams, M.D., Archidiacono, N., Rocchi, M., and Eichler, E.E. 2000. Molecular structure and evolution of an $\alpha$ satellite/non- $\alpha$ satellite junction at 16p11. Hum. Mol. Genet. 9: 113-123.

Horvath, J.E., Bailey, J.A., Locke, D.P., and Eichler, E.E. 2001. Lessons from the human genome: Transitions between euchromatin and heterochromatin. Hum. Mol. Genet. 10: 2215-2223.

Jackson, M. 2003. Duplicate, decouple, disperse: The evolutionary transience of human centromeric regions. Curr. Opin. Genet. Dev. 13: 629-635.

Ji, Y., Eichler, E.E., Schwartz, S., and Nicholls, R.D. 2000a. Structure of chromosomal duplicons and their role in mediating human genomic disorders. Genome Res. 10: 597-610.

Ji, Y., Rebert, N.A., Joslin, J.M., Higgins, M.J., Schultz, R.A., and Nicholls, R.D. 2000b. Structure of the highly conserved HERC2 gene and of multiple partially duplicated paralogs in human. Genome Res. 10: $319-329$.

Johnson, M.E., Viggiano, L., Bailey, J.A., Abdul-Rauf, M., Goodwin, G. Rocchi, M., and Eichler, E.E. 2001. Positive selection of a gene family during the emergence of humans and African apes. Nature 413: 514-519.

Karaman, M.W., Houck, M.L., Chemnick, L.G., Nagpal, S. Chawannakul, D., Sudano, D., Pike, B.L., Ho, V.V., Ryder, O.A., and Hacia, J.G. 2003. Comparative analysis of gene-expression patterns in human and African great ape cultured fibroblasts. Genome Res. 13: 1619-1630.

Kehrer-Sawatzki, H., Schreiner, B., Taenzer, S., Platzer, M., Muller, S., and Hameister, H. 2002. Molecular characterization of the pericentric inversion that causes differences between chimpanzee chromosome 19 and human chromosome 17. Am. J. Hum. Genet. 71: $375-388$.

Kehrer-Sawatzki, H., Sandig, C.A., Goidts, V., and Hameister, H. 2005a. Breakpoint analysis of the pericentric inversion between chimpanzee chromosome 10 and the homologous chromosome 12 in humans. Cytogenet. Genome Res. 108: 91-97.

Kehrer-Sawatzki, H., Sandig, C., Chuzhanova, N., Goidts, V., Szamalek, J.M., Tanzer, S., Muller, S., Platzer, M., Cooper, D.N., and Hameister, H. 2005b. Breakpoint analysis of the pericentric inversion distinguishing human chromosome 4 from the homologous chromosome in the chimpanzee (Pan troglodytes). Hum. Mutat. 25: $45-55$.

Locke, D.P., Archidiacono, N., Misceo, D., Cardone, M.F., Deschamps, S., Roe, B., Rocchi, M., and Eichler, E.E. 2003. Refinement of a chimpanzee pericentric inversion breakpoint to a segmental duplication cluster. Genome Biol. 4: R50.

Maresco, D.L., Blue, L.E., Culley, L.L., Kimberly, R.P., Anderson, C.L., and Theil, K.S. 1998. Localization of FCGR1 encoding Fc $\gamma$ receptor class I in primates: Molecular evidence for two pericentric inversions during the evolution of human chromosome 1. Cytogenet. Cell Genet. 82: $71-74$

Marques-Bonet, T., Caceres, M., Bertranpetit, J., Preuss, T.M., Thomas, J.Y., and Navarro, A. 2004. Chromosomal rearrangements and the genomic distribution of gene-expression divergence in humans and chimpanzees. Trends Genet. 20: 524-529.

Martin, J., Han, C., Gordon, L.A., Terry, A., Prabhakar, S., She, X., Xie, G., Hellsten, U., Chan, Y.M., Altherr, M., et al. 2004. The sequence and analysis of duplication-rich human chromosome 16. Nature 432: 988-994.

Misceo, D., Ventura, M., Eder, V., Rocchi, M., and Archidiacono, N. 2003. Human chromosome 16 conservation in primates.
Chromosome Res. 11: 323-326.

Montefalcone, G., Tempesta, S., Rocchi, M., and Archidiacono, N. 1999. Centromere repositioning. Genome Res. 9: 1184-1188.

Nahon, J.L. 2003. Birth of 'human-specific' genes during primate evolution. Genetica 118: 193-208.

Nickerson, E. and Nelson, D.L. 1998. Molecular definition of pericentric inversion breakpoints occurring during the evolution of humans and chimpanzees. Genomics 50: 368-372.

Panagopoulos, I., Aman, P., Fioretos, T., Hoglund, M., Johansson, B., Mandahl, N., Heim, S., Behrendtz, M., and Mitelman, F. 1994. Fusion of the FUS gene with ERG in acute myeloid leukemia with $\mathrm{t}(16 ; 21)(\mathrm{p} 11 ; \mathrm{q} 22)$. Genes Chrom. Cancer 11: 256-262.

Ross, M.T., LaBrie, S., McPherson, J., and Stanton, V.P. 1999. In Current protocols in human genetics. (eds. N.C. Dracopoli, et al.), pp. 5.6.15.6.5. Wiley, New York.

Samonte, R.V. and Eichler, E.E. 2002. Segmental duplications and the evolution of the primate genome. Nat. Rev. Genet. 3: 65-72.

Sharma, P., Watson, N., Robson, L., Gallo, J., and Smith, A. 1999. Novel chromosome 16 abnormality-der(16)del(16) $(\mathrm{q} 13) \mathrm{t}(16 ; 21)(\mathrm{p} 11.2 ; \mathrm{q} 22)$-Associated with acute myeloid leukemia. Cancer Genet. Cytogenet. 113: 25-28.

Shaw, C.J. and Lupski, J.R. 2004. Implications of human genome architecture for rearrangement-based disorders: The genomic basis of disease. Hum. Mol. Genet. 13: R57-64.

She, X., Jiang, Z., Clark, R.A., Liu, G., Cheng, Z., Tuzun, E., Church, D.M., Sutton, G., Halpern, A.L., and Eichler, E.E. 2004. Shotgun sequence assembly and recent segmental duplications within the human genome. Nature 431: 927-930.

Shimada, M.K., Kim, C.G., Kitano, T., Ferrell, R.E., Kohara, Y., and Saitou, N. 2005. Nucleotide sequence comparison of a chromosome rearrangement on human chromosome 12 and the corresponding ape chromosomes. Cytogenet. Genome Res. 108: 83-90.

Shing, D.C., Morley-Jacob, C.A., Roberts, I., Nacheva, E., and Coleman, N. 2002. Ewing's tumour: Novel recurrent chromosomal abnormalities demonstrated by molecular cytogenetic analysis of seven cell lines and one primary culture. Cytogenet. Genome Res. 97: $20-27$

Stankiewicz, P., Park, S.S., Inoue, K., and Lupski, J.R. 2001. The evolutionary chromosome translocation 4;19 in Gorilla gorilla is associated with microduplication of the chromosome fragment syntenic to sequences surrounding the human proximal CMT1A-REP. Genome Res. 11: 1205-1210.

Stankiewicz, P., Shaw, C.J., Withers, M., Inoue, K., and Lupski, J.R. 2004. Serial segmental duplications during primate evolution result in complex human genome architecture. Genome Res. 14: 2209-2220.

Taylor, J.S., Van de Peer, Y., and Meyer, A. 2001. Genome duplication, divergent resolution and speciation. Trends Genet. 17: 299-301.

Uddin, M., Wildman, D.E., Liu, G., Xu, W., Johnson, R.M, Hof, P.R., Kapatos, G., Grossman, L.I., and Goodman, M. 2004. Sister grouping of chimpanzees and humans as revealed by genome-wide phylogenetic analysis of brain gene expression profiles. Proc. Natl. Acad. Sci. 101: 2957-2962.

Ueda, C., Akasaka, T., Kurata, M., Maesako, Y., Nishikori, M. Ichinohasama, R., Imada, K., Uchiyama, T., and Ohno, H. 2002. The gene for interleukin-21 receptor is the partner of BCL6 in $\mathrm{t}(3 ; 16)(\mathrm{q} 27 ; \mathrm{p} 11)$, which is recurrently observed in diffuse large B-cell lymphoma. Oncogene 21: 368-376.

Vallender, E.J. and Lahn, B.T. 2004. Effects of chromosomal rearrangements on human-chimpanzee molecular evolution. Genomics 84: 757-761.

van Geel, M., Eichler, E.E., Beck, A.F., Shan, Z., Haaf, T., van der Maarel, S.M., Frants, R.R., and de Jong, P.J. 2002. A cascade of complex subtelomeric duplications during the evolution of the hominoid and Old World monkey genomes. Am. J. Hum. Genet. 70: 269-278.

Willeke, F., Ridder, R., Mechtersheimer, G., Schwarzbach, M., Duwe, A., Weitz, J., Lehnert, T., Herfarth, C., and von Knebel Doeberitz, M. 1998. Analysis of FUS-CHOP fusion transcripts in different types of soft tissue liposarcoma and their diagnostic implications. Clin. Cancer Res. 4: 1779-1784.

Won, Y.J. and Hey, J. 2005. Divergence population genetics of chimpanzees. Mol. Biol. Evol. 22: 297-307.

Wu, C.-I. and Ting, C.-T. 2004. Genes and speciation. Nat. Rev. Genet. 5: $114-122$.

Yamamoto, K., Nagata, K., Kida, A., and Hamaguchi, H. 2001. Deletion of $16 \mathrm{q} 11$ is a recurrent cytogenetic aberration in acute myeloblastic leukemia during disease progression. Cancer Genet. Cytogenet. 131: 65-68.

Yoder, A.D. and Yang, Z. 2000. Estimation of primate speciation dates using local molecular clocks. Mol. Biol. Evol. 17: 1081-1090.

Yunis, J.J. and Prakash, O. 1982. The origin of man: A chromosomal pictorial legacy. Science 215: 1525-1530. 


\section{Goidts et al.}

Zhang, J., Wang, X., and Podlaha, O. 2004. Testing the chromosomal speciation hypothesis for humans and chimpanzees. Genome Res. 14: $845-851$.

\section{Web site references}

http://www.ecacc.org.uk/; The European Collection of Cell Cultures site. http://www.invitrogen.com/; Invitrogen home page.

http://www.chori.org/bacpac/; Children's Hospital Oakland-BAC-PAC Resources. http://www.ncbi.nlm.nih.gov/blast/; National Center for Biotechnology Information (NCBI).

http://www.ensembl.org/; Ensembl, a joint project between EMBL-EBI and the Sanger Institute that generates automatic annotation for eukaryotic genomes.

http://projects.tcag.ca/humandup/; human genome segmental duplication database.

http://www.ncbi.nlm.nih.gov/; National Center for Biotechnology Information.

Received January 21, 2005; accepted in revised form April 5, 2005. 


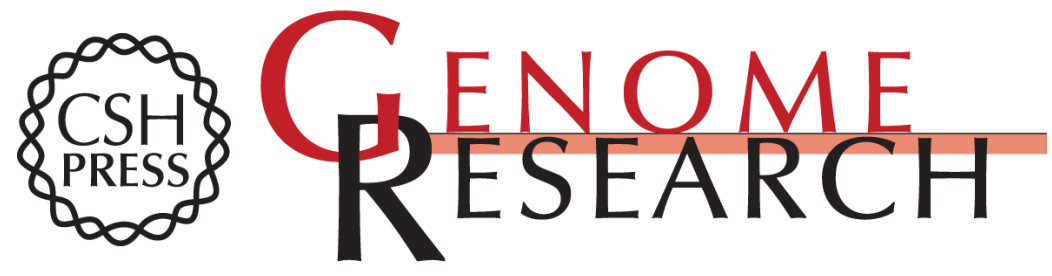

\section{Independent intrachromosomal recombination events underlie the pericentric inversions of chimpanzee and gorilla chromosomes homologous to human chromosome 16}

Violaine Goidts, Justyna M. Szamalek, Pieter J. de Jong, et al.

Genome Res. 2005 15: 1232-1242

Access the most recent version at doi:10.1101/gr.3732505

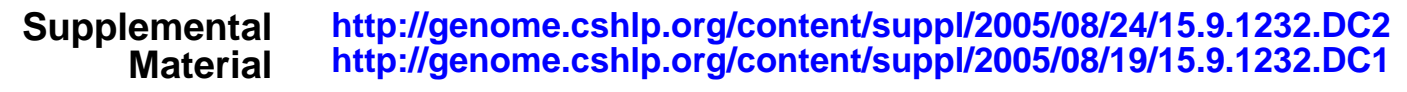

References This article cites 55 articles, 16 of which can be accessed free at:

http://genome.cshlp.org/content/15/9/1232.full.html\#ref-list-1

\section{License}

Email Alerting

Receive free email alerts when new articles cite this article - sign up in the box at the Service top right corner of the article or click here.

\section{Affordable, Accurate Sequencing.}

